\title{
An Iterative Shrinking Projection Method for Solving Fixed Point Problems of Closed and $\phi$-Quasi-Strict Pseudocontractions along with Generalized Mixed Equilibrium Problems in Banach Spaces
}

\author{
Kasamsuk Ungchittrakool ${ }^{1,2}$ \\ ${ }^{1}$ Department of Mathematics, Faculty of Science, Naresuan University, Phitsanulok 65000, Thailand \\ ${ }^{2}$ Centre of Excellence in Mathematics, CHE, Si Ayutthaya Road, Bangkok 10400, Thailand
}

Correspondence should be addressed to Kasamsuk Ungchittrakool, kasamsuku@nu.ac.th

Received 22 April 2012; Revised 4 July 2012; Accepted 29 July 2012

Academic Editor: Simeon Reich

Copyright (C 2012 Kasamsuk Ungchittrakool. This is an open access article distributed under the Creative Commons Attribution License, which permits unrestricted use, distribution, and reproduction in any medium, provided the original work is properly cited.

We provide some new type of mappings associated with pseudocontractions by introducing some actual examples in smooth and strictly convex Banach spaces. Moreover, we also find the significant inequality related to the mappings mentioned in the paper and the mappings defined from generalized mixed equilibrium problems on Banach spaces. We propose an iterative shrinking projection method for finding a common solution of generalized mixed equilibrium problems and fixed point problems of closed and $\phi$-quasi-strict pseudo-contractions. Our results hold in reflexive, strictly convex, and smooth Banach spaces with the property $(K)$. The results of this paper improve and extend the corresponding results of Zhou and Gao (2010) and many others.

\section{Introduction}

It is well known that, in an infinite-dimensional Hilbert space, the normal Mann's iterative algorithm [1] has only weak convergence, in general, even for nonexpansive mappings [2,3]. Consequently, in order to obtain strong convergence, Nakajo and Takahashi [4] modified the normal Mann's iteration algorithm, later well known as a hybrid projection iteration method. Since then, the hybrid method has undergone rapid developments. For the details, the readers are referred to papers [5-9] and the references therein. 
Let $E$ be a smooth Banach space, and let $E^{*}$ be the dual of $E$. The function $\phi: E \times E \rightarrow \mathbb{R}$ is defined by

$$
\phi(y, x)=\|y\|^{2}-2\langle y, J x\rangle+\|x\|^{2}
$$

for all $x, y \in E$, which was studied by Alber [10], Kamimura and Takahashi [11], and Reich [12], where $J$ is the normalized duality mapping from $E$ to $2^{E^{*}}$ defined by

$$
J(x)=\left\{f \in E^{*}:\langle x, f\rangle=\|x\|^{2}=\|f\|^{2}\right\}
$$

where $\langle\cdot, \cdot\rangle$ denotes the duality paring. It is well known that if $E$ is smooth, then $J$ is single valued and if $E$ is strictly convex, then $J$ is injective (one to one). Let $C$ be a closed convex subset of $E$, and let $T$ be a mapping from $C$ into itself. We denote by $F(T)$ the set of fixed points of $T$. A point $p$ in $C$ is said to be an asymptotic fixed point of $T$ [12] if $C$ contains a sequence $\left\{x_{n}\right\}$, which converges weakly to $p$ such that the strong $\lim _{n \rightarrow \infty}\left(x_{n}-T x_{n}\right)=0$. The set of asymptotic fixed points of $T$ will be denoted by $\widehat{F}(T)$. A mapping $T$ from $C$ into itself is called nonexpansive if $\|T x-T y\| \leqslant\|x-y\|$ for all $x, y \in C$ and relatively nonexpansive [13-16] if $\widehat{F}(T)=F(T)$ and $\phi(p, T x) \leqslant \phi(p, x)$ for all $x \in C$ and $p \in F(T)$. The asymptotic behavior of relatively nonexpansive mapping was studied in [13-16].

In 2005, Matsushita and Takahashi [16] proposed the hybrid iteration method with generalized projection for relatively nonexpansive mapping $T$ in the framework of uniformly smooth and uniformly convex Banach spaces $E$ as follows:

$$
\begin{gathered}
x_{0} \in C \text { chosen arbitrarily, } \\
y_{n}=J^{-1}\left(\alpha_{n} J x_{n}+\left(1-\alpha_{n}\right) J T x_{n}\right), \\
C_{n}=\left\{z \in C: \phi\left(z, y_{n}\right) \leq \phi\left(z, x_{n}\right)\right\}, \\
Q_{n}=\left\{z \in C:\left\langle x_{n}-z, J x_{0}-J x_{n}\right\rangle \geq 0\right\}, \\
x_{n+1}=\prod_{C_{n} \cap Q_{n}}\left(x_{0}\right),
\end{gathered}
$$

where $J$ is the duality mapping on $E$ and $\Pi_{C}(\cdot)$ is the generalized projection from $E$ onto a nonempty closed convex subset $C$. Based on the guidelines of Matsushita and Takahashi [16], Plubtieng and Ungchittrakool [17, 18] studied and developed (1.3) to the case of two relatively nonexpansive mappings and finite family of relatively nonexpansive mappings, respectively.

On the other hand, for a real Banach space $E$ and the dual $E^{*}$, let $C$ be a nonempty closed convex subset of $E$. Let $\Theta: C \times C \rightarrow \mathbb{R}$ a bifunction, $\varphi: C \rightarrow \mathbb{R}$ a real-valued function, and $A: C \rightarrow E^{*}$ be a nonlinear mapping. The generalized mixed equilibrium problem is to find $x \in C$ such that

$$
\Theta(x, y)+\langle A x, y-x\rangle+\varphi(y)-\varphi(x) \geq 0, \quad \forall y \in C
$$


The solution set of (1.4) is denoted by $\operatorname{GMEP}(\Theta, A, \varphi)$, that is,

$$
\operatorname{GMEP}(\Theta, A, \varphi)=\{x \in C: \Theta(x, y)+\langle A x, y-x\rangle+\varphi(y)-\varphi(x) \geq 0, \forall y \in C\}
$$

If $A=0$, the problem (1.4) reduces to the mixed equilibrium problem for $\Theta$, denoted by $\operatorname{MEP}(\Theta, \varphi)$, which is to find $x \in C$ such that

$$
\Theta(x, y)+\varphi(y)-\varphi(x) \geq 0, \quad \forall y \in C
$$

If $\Theta=0$, the problem (1.4) reduces to the mixed variational inequality of Browder type, denoted by $\operatorname{VI}(C, A, \varphi)$, which is to find $x \in C$ such that

$$
\langle A x, y-x\rangle+\varphi(y)-\varphi(x) \geq 0, \quad \forall y \in C
$$

If $A=0$ and $\varphi=0$, the problem (1.4) reduces to the equilibrium problem for $\Theta$ (for short, $\mathrm{EP})$, denoted by $\mathrm{EP}(\Theta)$, which is to find $x \in C$ such that

$$
\Theta(x, y) \geq 0, \quad \forall y \in C
$$

If $\Theta=0$ and $A=0$, the problem (1.4) reduces to the minimization problem for $\varphi$, denoted by $\operatorname{Argmin}(\varphi)$, which is to find $x \in C$ such that

$$
\varphi(x) \leq \varphi(y), \quad \forall y \in C
$$

The above formulation (1.7) was shown in [19] to cover monotone inclusion problems, saddle point problems, variational inequality problems, minimization problems, optimization problems, vector equilibrium problems, and Nash equilibria in noncooperative games. In addition, there are several other problems, for example, the complementarity problem, fixed point problem and optimization problem, which can also be written in the form of (1.8). However, (1.4) is very general, it covers the problems mentioned above as special cases.

In 2007, S. Takahashi and W. Takahashi [20] and Tada and Takahashi [21, 22] proved weak and strong convergence theorems for finding a common element of the set of solution of an equilibrium problem (1.8) and the set of fixed points of a nonexpansive mapping in a Hilbert space. Takahashi et al. [9] studied a strong convergence theorem by the hybrid method for a family of nonexpansive mappings in Hilbert spaces as follows: $x_{0} \in H, C_{1}=C$ and $x_{1}=P_{C_{1}} x_{0}$, and let

$$
\begin{gathered}
y_{n}=\alpha_{n} x_{n}+\left(1-\alpha_{n}\right) T_{n} x_{n}, \\
C_{n+1}=\left\{z \in C_{n}:\left\|y_{n}-z\right\| \leq\left\|x_{n}-z\right\|\right\}, \\
x_{n+1}=P_{C_{n+1}} x_{0}, \quad n \in \mathbb{N},
\end{gathered}
$$

where $0 \leqslant \alpha_{n} \leqslant a<1$ for all $n \in \mathbb{N}$ and $\left\{T_{n}\right\}$ is a sequence of nonexpansive mappings of $C$ into itself such that $\bigcap_{n=1}^{\infty} F\left(T_{n}\right) \neq \emptyset$. They proved that if $\left\{T_{n}\right\}$ satisfies some appropriate conditions, then $\left\{x_{n}\right\}$ converges strongly to $P_{\bigcap_{n=1}^{\infty} F\left(T_{n}\right)} x_{0}$. 
Motivated by Takahashi et al. [9], Takahashi and Zembayashi [23] (see also [24]) introduced and proved a hybrid projection algorithm for solving equilibrium problems and fixed point problems of a relatively nonexpansive mapping $S$ in the framework of uniformly smooth and uniformly convex Banach space as follows:

$$
\begin{gathered}
x_{0}=x, \quad C_{0}=C, \\
y_{n}=J^{-1}\left(\alpha_{n} J x_{n}+\left(1-\alpha_{n}\right) J S x_{n}\right), \\
u_{n} \in C \text { such that } \Theta\left(u_{n}, y\right)+\frac{1}{r_{n}}\left\langle y-u_{n} J u_{n}-J y_{n}\right\rangle \geq 0, \quad \forall y \in C, \\
C_{n+1}=\left\{z \in C_{n}: \phi\left(z, u_{n}\right) \leq \phi\left(z, x_{n}\right)\right\}, \\
x_{n+1}=\prod_{C_{n+1}} x,
\end{gathered}
$$

where $\Pi_{C_{n+1}}(\cdot)$ is the generalized projection from $E$ onto $C_{n+1}$. Under some appropriate assumptions on $\Theta,\left\{\alpha_{n}\right\}$, and $\left\{r_{n}\right\}$, they proved that the sequence $\left\{x_{n}\right\}$ converges strongly to $\prod_{F(S) \cap E P(\Theta)}\left(x_{0}\right)$.

In 2010, Zhou and Gao [25] introduced the definition of a quasi-strict pseudocontraction related to the function $\phi$ and proved a hybrid projection algorithm for finding a fixed point of a closed and quasi-strict pseudocontraction in more general framework than uniformly smooth and uniformly convex Banach spaces as follows:

$$
\begin{aligned}
& x_{0} \in E, \text { chosen arbitrarily, } \\
& C_{1}=C, \\
& x_{1}=\Pi_{C_{1}}\left(x_{0}\right), \\
& u_{n} \in C \text { such that } \\
& C_{n+1}=\left\{z \in C_{n}: \phi\left(x_{n}, T x_{n}\right) \leq \frac{2}{1-k}\left\langle x_{n}-z, J x_{n}-J T x_{n}\right\rangle\right\}, \\
& x_{n+1}=\prod_{C_{n+1}}\left(x_{0}\right),
\end{aligned}
$$

where $\Pi_{C_{n+1}}$ is the generalized projection from $E$ onto $C_{n+1}$. They proved that the sequence $\left\{x_{n}\right\}$ converges strongly to $\Pi_{F(T)}\left(x_{0}\right)$.

Motivated and inspired by the above research work, in this paper, we provide some new type of mappings associated with pseudocontractions by introducing some actual examples in smooth and strictly convex Banach spaces. Furthermore, by employing the inequality that appeared in Lemma 2.10 together with (1.12) and some facts of Zhou and Gao [25], we create an iterative shrinking projection method for finding a common solution of generalized mixed equilibrium problems and fixed point problems of closed and $\phi$ quasi-strict pseudocontractions in the framework of reflexive, strictly convex, and smooth Banach spaces with the property $(K)$. The results of this research improve and extend the corresponding results of Zhou and Gao [25] and many others. 


\section{Preliminaries}

In this paper, we denote by $E$ and $E^{*}$ a real Banach space and the dual space of $E$, respectively. Let $C$ be a nonempty closed convex subset of $E$. We denote by $J$ the normalized duality mapping from $E$ to $2^{E^{*}}$ defined by (1.1). Let $S(E):=\{x \in E:\|x\|=1\}$ be the unit sphere of $E$. Then, a Banach space $E$ is said to be strictly convex $\|(x+y) / 2\|<1$ for all $x, y \in S(E)$ and $x \neq y$. It is also said to be uniformly convex if $\lim _{n \rightarrow \infty}\left\|x_{n}-y_{n}\right\|=0$ for any two sequences $\left\{x_{n}\right\}$ and $\left\{y_{n}\right\}$ in $S(E)$ such that $\lim _{n \rightarrow \infty}\left\|\left(x_{n}+y_{n}\right) / 2\right\|=1$. The Banach space $E$ is said to be smooth provided

$$
\lim _{t \rightarrow 0} \frac{\|x+t y\|-\|x\|}{t}
$$

exists for each $x, y \in S(E)$. In this case, the norm of $E$ is said to be Gateaux differentiable. The norm of $E$ is said to be Fréchet differentiable if for each $x \in S(E)$, the limit (2.1) is attained uniformly for $y \in S(E)$. The norm of $E$ is said to be uniformly Fréchet differentiable (and $E$ is said to be uniformly smooth) if the limit (2.1) is attained uniformly for $x, y \in S(E)$.

A Banach space $E$ is said to have the property (K) (or Kadec-Klee property) if for any sequence $\left\{x_{n}\right\} \subset E$ such that $x_{n} \rightarrow x$ weakly and $\left\|x_{n}\right\| \rightarrow\|x\|$, then $\left\|x_{n}-x\right\| \rightarrow 0$. For more information concerning property $(K)$ the reader is referred to [26] and references cited there.

A mapping $T: C \rightarrow C$ is said to be closed if for any sequence $\left\{x_{n}\right\} \subset C$ with $x_{n} \rightarrow x$ and $T x_{n} \rightarrow y, x=y$.

We also know the following properties (see [27-29] for details):

(i) if $E$ is smooth ( $\Leftrightarrow E^{*}$ is strictly convex), then $J$ is single-valued,

(ii) if $E$ is strictly convex ( $\Leftrightarrow E^{*}$ is smooth), then $J$ is one-to-one (i.e., $J(x) \cap J(y)=\emptyset$ for all $x \neq y)$,

(iii) if $E$ is reflexive ( $\Leftrightarrow E^{*}$ is reflexive), then $J$ is surjective,

(iv) if $E^{*}$ is smooth and reflexive, then $J^{-1}: E^{*} \rightarrow 2^{E}$ is single-valued and demicontinuous (i.e., if $\left\{x_{n}^{*}\right\} \subset E^{*}$ such that $x_{n}^{*} \rightarrow x^{*}$, then $\left.J^{-1}\left(x_{n}^{*}\right) \rightarrow J^{-1}\left(x^{*}\right)\right)$,

(v) $E$ is uniformly smooth if and only if $E^{*}$ is uniformly convex,

(vi) if $E$ is uniformly convex, then

(a) it is strictly convex,

(b) it is reflexive,

(c) it satisfies the property $(K)$,

(vii) if $E$ is a Hilbert space, then $J$ is the identity operator.

It is also very well known that if $C$ is a nonempty closed convex subset of a Hilbert space $H$ and $P_{C}: H \rightarrow C$ is the metric projection of $H$ onto $C$, then $P_{C}$ is nonexpansive. This fact actually characterizes Hilbert spaces, and, consequently, it is not available in more general Banach spaces. In this connection, Alber [10] recently introduced a generalized projection operator $\Pi_{C}$ in a Banach space $E$ that is an analogue of the metric projection in Hilbert spaces.

Let $C$ be a nonempty closed convex subset of a smooth, strictly convex, and reflexive Banach space $E$, for any $x \in E$. The generalized projection $\Pi_{C}: E \rightarrow C$ is a map that assigns to 
an arbitrary point $x \in E$ the minimum point of the functional $\phi(x, y)$, that is, $\Pi_{C} x=\bar{x}$, where $\bar{x}$ is the solution to the minimization problem

$$
\phi(\bar{x}, x)=\min _{y \in C} \phi(y, x)
$$

Existence and uniqueness of the operator $\Pi_{C}$ follow from the properties of the functional $\phi(x, y)$ and strict monotonicity of the mapping $J$ (see, e.g., $[10,11,27,29,30])$. In Hilbert spaces, $\phi(y, x)=\|y-x\|^{2}$ and $\Pi_{C}$ coincides with the metric projection $P_{C}$.

It is obvious from the definition of function $\phi$ that

$$
\begin{gathered}
(\|y\|-\|x\|)^{2} \leq \phi(y, x) \leq(\|y\|+\|x\|)^{2}, \quad \forall x, y \in E, \\
\phi(x, y)=\phi(x, z)+\phi(z, y)+2\langle x-z, J z-J y\rangle, \quad \forall x, y, z \in E .
\end{gathered}
$$

Remark 2.1. If $E$ is a reflexive strictly convex and smooth Banach space, then, for $x, y \in$ $E, \phi(x, y)=0$ if and only if $x=y$. It is sufficient to show that if $\phi(x, y)=0$, then $x=y$. From (2.3), we have $\|x\|=\|y\|$. This implies $\langle x, J y\rangle=\|x\|^{2}=\|J y\|^{2}$. From the definitions of $J$, we have $J x=J y$. That is, $x=y$; one may consult $[27,29]$ for the details.

$T$ is said to be a $\phi$-quasi-strict pseudocontraction [25, page 230] if there exists a constant $k \in[0,1)$ and $F(T) \neq \emptyset$ such that $\phi(p, T x) \leq \phi(p, x)+k \phi(x, T x)$ for all $x \in C$ and $p \in F(T)$. In particular, $T$ is said to be $\phi$-quasi-nonexpansive if $k=0$ and $T$ is said to be $\phi$-quasi-pseudocontractive if $k=1$.

Let $C$ be a nonempty closed convex subset of a real Banach space $E$ with the dual $E^{*}$. A mapping $A: D(A) \subset E \rightarrow E^{*}$ is said to be monotone if, for each $x, y \in D(A)$, the following inequality holds:

$$
\langle x-y, A x-A y\rangle \geqslant 0
$$

$A$ is said to be $r$-inverse strongly monotone if there exists a positive real number $r$ such that

$$
\langle x-y, A x-A y\rangle \geq r\|A x-A y\|_{E^{* \prime}}^{2} \quad \forall x, y \in D(A) .
$$

$A$ is said to be $r$-quasi inverse strongly monotone if $A^{-1}(0)=\{z \in D(A): A z=0\} \neq \emptyset$ and there exists a positive real number $r$ such that for each $u \in A^{-1}(0)$, the following inequality holds:

$$
\langle x-u, A x\rangle \geqslant r\|A x\|_{E^{*}}^{2}, \quad \forall x \in D(A) .
$$

Without loss of generality we can assume that the constant $r \in(0,1 / 2]$ since if $A$ is $\hat{r}$-quasi inverse strongly monotone (or $\widehat{r}$-inverse strongly monotone), then we can find $r \in(0,1 / 2]$ such that $\hat{r} \geq r$ and then $\widehat{r}\|A x\|_{E^{*}}^{2} \geq r\|A x\|_{E^{*}}^{2}\left(\right.$ or $\left.\widehat{r}\|A x-A y\|_{E^{*}}^{2} \geq r\|A x-A y\|_{E^{*}}^{2}\right)$ for all $\hat{r}>0$ (i.e., $A$ is $r$-quasi inverse strongly monotone).

The following is the example of $\phi$-quasi-strict pseudocontractions in the framework of a smooth and strictly convex Banach space $E$. 
Example 2.2. Let $E$ be a smooth and strictly convex Banach space $E$ with dual $E^{*}$ and $J$ a normalized duality mapping. Let $\alpha \in[0,1]$, and define the mapping $A_{\alpha}: E \rightarrow E$ by

$$
A_{\alpha}(x)=\alpha x, \quad \forall x \in E
$$

It follows that the mapping $J A_{\alpha}: E \rightarrow E^{*}$ with the set of zero points is given as

$$
\begin{aligned}
\left(J A_{\alpha}\right)^{-1}(0) & =\left\{z \in E: 0=J A_{\alpha}(z)=J(\alpha z)=\alpha J z\right\} \\
& = \begin{cases}\{0\}, & \text { if } 0<\alpha \leq 1 \\
E, & \text { if } \alpha=0\end{cases}
\end{aligned}
$$

Thus for each $u \in\left(J A_{\alpha}\right)^{-1}(0)$ and $r \in(0,1 / 2]$ if $0<\alpha \leq 1$, we obtain

$$
\begin{aligned}
\left\langle x-u, J A_{\alpha} x\right\rangle & =\left\langle x-0, J A_{\alpha} x\right\rangle=\langle x, J(\alpha x)\rangle=\langle x, \alpha J(x)\rangle=\alpha\|x\|^{2} \\
& \geq \alpha^{2}\|x\|^{2}=\langle\alpha x, J(\alpha x)\rangle=\left\langle A_{\alpha} x, J\left(A_{\alpha} x\right)\right\rangle=\left\|A_{\alpha} x\right\|^{2} \\
& =\left\|J A_{\alpha} x\right\|_{E^{*}}^{2} \\
& \geq r\left\|J A_{\alpha} x\right\|_{E^{*}}^{2}, \quad \forall x \in E .
\end{aligned}
$$

On the other hand, if $\alpha=0$, we have $\left\langle x-u, J A_{\alpha} x\right\rangle=0=r\|0\|_{E^{*}}^{2}=r\left\|J A_{\alpha} x\right\|_{E^{*}}^{2}$, for all $x \in E$. Therefore, $J A_{\alpha}$ is $r$-quasi inverse strongly monotone. So, we can define the following set:

$$
Q=\left\{J \tilde{A}: E \rightarrow E^{*} \mid\langle x-u, J \tilde{A} x\rangle \geq r\|J \tilde{A} x\|_{E^{*^{\prime}}}^{2}, \forall x \in E, u \in(J \tilde{A})^{-1}(0)\right\},
$$

where $\tilde{A}: E \rightarrow E$ and $r \in(0,1 / 2]$ (note that $J A_{\alpha} \in Q \neq \emptyset$ ). In the same way, let $\widehat{A}: E \rightarrow E$, and define

$$
\mathcal{L}=\{J \widehat{A} \in \mathcal{Q} \mid J(x-\widehat{A} x)=J x-J \widehat{A} x,\langle x, J \widehat{A} x\rangle=\langle\widehat{A} x, J x\rangle, \forall x \in E\} .
$$

We claim that $J A_{\alpha} \in \mathcal{L}$. Note that $J A_{\alpha} \in \mathcal{Q}$, and let us consider

$$
\begin{aligned}
J\left(x-A_{\alpha} x\right) & =J(x-\alpha x)=(1-\alpha) J x=J x-\alpha J x=J x-J(\alpha x) \\
& =J x-J A_{\alpha} x .
\end{aligned}
$$

Furthermore, it is also found that

$$
\left\langle x, J A_{\alpha} x\right\rangle=\langle x, J(\alpha x)\rangle=\langle\alpha x, J x\rangle=\left\langle A_{\alpha} x, J x\right\rangle .
$$

This means that $J A_{\alpha} \in \mathcal{L} \neq \emptyset$. So, we have the claim. 
Now, we pick arbitrarily $J \widehat{A} \in \mathcal{L}$ and claim that $(I-\widehat{A}): E \rightarrow E$ is a $\phi$-quasi-strict pseudocontraction (i.e., $\exists k \in[0,1)$ s.t. $\phi(q,(I-\widehat{A}) x) \leqslant \phi(q, x)+k \phi(x,(I-\widehat{A}) x)$ for all $x \in E$ and $q \in F(I-\widehat{A}))$.

Since $J \widehat{A} \in \mathcal{L}$, we have

$$
\begin{aligned}
\|\widehat{A} x\|^{2} & =\langle\widehat{A} x, J \widehat{A} x\rangle=\langle x-(I-\widehat{A}) x, J x-(J x-J \widehat{A} x)\rangle \\
& =\langle x-(I-\widehat{A}) x, J x\rangle-\langle x-(I-\widehat{A}) x, J(I-\widehat{A}) x\rangle \\
& =\|x\|^{2}-\langle(I-\widehat{A}) x, J x\rangle-\langle x, J(I-\widehat{A}) x\rangle+\|(I-\widehat{A}) x\|^{2} \\
& =\left(\|x\|^{2}-2\langle x, J(I-\widehat{A}) x\rangle+\|(I-\widehat{A}) x\|^{2}\right)+\langle x, J(I-\widehat{A}) x\rangle-\langle(I-\widehat{A}) x, J x\rangle \\
& =\phi(x,(I-\widehat{A}) x)+\langle x, J x\rangle-\langle x, J \widehat{A} x\rangle-\langle x, J x\rangle+\langle\widehat{A} x, J x\rangle \\
& =\phi(x,(I-\widehat{A}) x) .
\end{aligned}
$$

Note that $\mathcal{L} \subset \mathcal{Q}$, and thus $(J \widehat{A})^{-1}(0) \neq \emptyset$. It is not difficult to check that $(J \widehat{A})^{-1}(0)=$ $F(I-\widehat{A})$. Therefore, for each $q \in F(I-\widehat{A})$, we have

$$
\begin{aligned}
\phi(q,(I-\widehat{A}) x) & =\|q\|^{2}-2\langle q, J(I-\widehat{A}) x\rangle+\|(I-\widehat{A}) x\|^{2} \\
& =\|q\|^{2}-2\langle q, J x-J \widehat{A} x\rangle+\|(I-\widehat{A}) x\|^{2} \\
& =\left(\|q\|^{2}-2\langle q, J x\rangle+\|x\|^{2}\right)-\|x\|^{2}+2\langle q, J \widehat{A} x\rangle+\|(I-\widehat{A}) x\|^{2} \\
& =\phi(q, x)-\|x\|^{2}-2\langle x-q, J \widehat{A} x\rangle+2\langle x, J \widehat{A} x\rangle+\|(I-\widehat{A}) x\|^{2} \\
& \leq \phi(q, x)-\|x\|^{2}-2 r\|J \widehat{A} x\|^{2}+2\langle x, J x\rangle-2\langle x, J x-J \widehat{A} x\rangle+\|(I-\widehat{A}) x\|^{2} \\
& =\phi(q, x)-2 r\|\widehat{A} x\|^{2}+\left(\|x\|^{2}-2\langle x, J(I-\widehat{A}) x\rangle+\|(I-\widehat{A}) x\|^{2}\right) \\
& =\phi(q, x)-2 r \phi(x,(I-\widehat{A}) x)+\phi(x,(I-\widehat{A}) x) \\
& =\phi(q, x)+(1-2 r) \phi(x,(I-\widehat{A}) x) \\
& =\phi(q, x)+k \phi(x,(I-\widehat{A}) x),
\end{aligned}
$$

where $k:=(1-2 r)$. So, we obtain the desired result. 
Next, we claim that the mapping $\left(I-A_{\alpha}\right)$ as mentioned previously is closed. Let $\left\{x_{n}\right\} \subset$ $E$ such that $x_{n} \rightarrow x$; then we have

$$
\begin{aligned}
\left(I-A_{\alpha}\right) x_{n} & =x_{n}-A_{\alpha} x_{n}=x_{n}-\alpha x_{n}=(1-\alpha) x_{n} \\
& \longrightarrow(1-\alpha) x \quad \text { as } n \longrightarrow \infty
\end{aligned}
$$

Moreover, $\left(I-A_{\alpha}\right) x=x-A_{\alpha} x=x-\alpha x=(1-\alpha) x$. This shows that $\left(I-A_{\alpha}\right)$ is a closed mapping.

Remark 2.3. A relatively nonexpansive mapping is a $\phi$-quasi-strict pseudocontraction, but the converse may be not true.

Example 2.4. Let $E$ be a reflexive, strictly convex, and smooth Banach space. Let $A \subset E \times E^{*}$ be a maximal monotone mapping such that $A^{-1}(0)$ is nonempty. Then, $J_{r}=(J+r A)^{-1} J$ is a closed and $\phi$-quasi-strict pseudocontraction from $E$ onto $D(A)$ with constant $k=0$ and $F\left(J_{r}\right)=A^{-1}(0)$.

Example 2.5. Let $\Pi_{C}$ be the generalized projection from a smooth, strictly convex, and reflexive Banach space $E$ onto a nonempty closed convex subset $C$ of $E$. Then, $\Pi_{C}$ is a closed and $\phi$-quasi-strict pseudocontraction from $E$ onto $C$ with constant $k=0$ and $F\left(\Pi_{C}\right)=C$.

For solving the equilibrium problem for a bifunction $\Theta: C \times C \rightarrow \mathbb{R}$, let us assume that $\Theta$ satisfies the following conditions:

(A1) $\Theta(x, x)=0$ for all $x \in C$,

(A2) $\Theta$ is monotone, that is, $\Theta(x, y)+\Theta(y, x) \leq 0$ for all $x, y \in C$,

(A3) for each $x, y, z \in C$,

$$
\lim _{t \downarrow 0} \Theta(t z+(1-t) x, y) \leq \Theta(x, y),
$$

(A4) for each $x \in C, y \mapsto \Theta(x, y)$ is convex and lower semicontinuous.

Lemma 2.6 (Blum and Oettli [19]). Let $C$ be a nonempty closed convex subset of a smooth, strictly convex, and reflexive Banach space $E$, and let $\Theta$ be a bifunction of $C \times C$ into $\mathbb{R}$ satisfying (A1)-(A4). Let $r>0$ and $x \in E$. Then, there exists $z \in C$ such that

$$
\Theta(z, y)+\frac{1}{r}\langle y-z, J z-J x\rangle \geq 0, \quad \forall y \in C
$$


Lemma 2.7 (Takahashi and Zembayashi [24]). Let $C$ be a closed convex subset of a uniformly smooth, strictly convex, and reflexive Banach space $E$, and let $\Theta$ be a bifunction from $C \times C$ to $\mathbb{R}$ satisfying (A1)-(A4). For $r>0$ and $x \in E$, define a mapping $T_{r}: E \rightarrow C$ as follows:

$$
T_{r} x=\left\{z \in C: \Theta(z, y)+\frac{1}{r}\langle y-z, J z-J x\rangle \geq 0, \forall y \in C\right\}
$$

for all $x \in C$. Then, the following hold:

(i) $T_{r}$ is single valued,

(ii) $T_{r}$ is firmly nonexpansive-type mapping, that is, for any $x, y \in H$,

$$
\left\langle T_{r} x-T_{r} y, J T_{r} x-J T_{r} y\right\rangle \leq\left\langle T_{r} x-T_{r} y, J x-J y\right\rangle,
$$

(iii) $F\left(T_{r}\right)=\mathrm{EP}(\Theta)$,

(iv) $\mathrm{EP}(\Theta)$ is closed and convex

Lemma 2.8 (Takahashi and Zembayashi [24]). Let $C$ be a closed convex subset of a smooth, strictly convex and reflexive Banach space $E$, let $\Theta$ be a bifunction from $C \times C$ to $\mathbb{R}$ satisfying (A1)-(A4), and let $r>0$. Then, for $x \in E$ and $q \in F\left(T_{r}\right)$,

$$
\phi\left(p, T_{r} x\right)+\phi\left(T_{r} x, x\right) \leq \phi(p, x) .
$$

Lemma 2.9 (Zhang [31]). Let $C$ be a closed convex subset of a smooth, strictly convex, and reflexive Banach space $E$. Let $A: C \rightarrow E^{*}$ be a continuous and monotone mapping, $\varphi: C \rightarrow \mathbb{R}$ a lower semi-continuous and convex function, and $\Theta$ a bifunction from $C \times C$ to $\mathbb{R}$ satisfying (A1)-(A4). For $r>0$ and $x \in E$, there exists $u \in C$ such that

$$
\Theta(u, y)+\langle A u, y-u\rangle+\varphi(y)-\varphi(u)+\frac{1}{r}\langle y-u, J u-J x\rangle \geq 0, \quad \forall y \in C .
$$

Define a mapping $K_{r}: C \rightarrow C$ as follows:

$$
K_{r}(x)=\left\{u \in C: \Theta(u, y)+\langle A u, y-u\rangle+\varphi(y)-\varphi(u)+\frac{1}{r}\langle y-u, J u-J x\rangle \geq 0, \forall y \in C\right\}
$$

for all $x \in C$. Then, the following conclusions hold:

(1) $K_{r}$ is single valued,

(2) $K_{r}$ is firmly nonexpansive type, that is, for all $x, y \in E$,

$$
\left\langle K_{r} x-K_{r} y, J K_{r} x-J K_{r} y\right\rangle \leq\left\langle K_{r} x-K_{r} y, J x-J y\right\rangle,
$$

(3) $F\left(K_{r}\right)=\operatorname{GMEP}(\Theta, A, \varphi)$,

(4) $\operatorname{GMEP}(\Theta, A, \varphi)$ is closed and convex,

(5) $\phi\left(p, K_{r} x\right)+\phi\left(K_{r} x, x\right) \leq \phi(p, x) \forall p \in F\left(K_{r}\right), x \in E$.

The following lemmas are crucial for the proofs of the main results in this paper. 
Lemma 2.10. Let $E$ be a reflexive, strictly convex, and smooth Banach space. Assume that $C$ is a nonempty closed convex subset of $E$. Let $T: C \rightarrow C$ be a $\phi$-quasi-strict pseudocontraction, and let $K_{r}: C \rightarrow C$ be as in Lemma 2.9 such that $\Omega:=F(T) \cap \operatorname{GMEP}(\Theta, A, \varphi) \neq \emptyset$. Then

$$
\phi\left(x, K_{r} T x\right)+\phi\left(K_{r} T x, T x\right) \leq \frac{2}{1-k}\langle x-p, J x-J T x\rangle+2\left\langle x-p, J T x-J K_{r} T x\right\rangle
$$

for all $x \in C$ and $p \in \Omega$.

Proof. Let $x \in C$ and $p \in \Omega$. By the $\phi$-quasi-strict pseudocontractility of $T$ and (2.4) we have

$$
\begin{aligned}
\phi(p, T x) & \leq \phi(p, x)+k \phi(x, T x) \\
& \Leftrightarrow \phi(p, x)+\phi(x, T x)+2\langle p-x, J x-J T x\rangle \leq \phi(p, x)+k \phi(x, T x) \\
& \Leftrightarrow \phi(x, T x) \leq \frac{2}{1-k}\langle x-p, J x-J T x\rangle .
\end{aligned}
$$

It follows from (2.4), Lemma 2.9 (5), and (2.27) that

$$
\begin{aligned}
\phi(p, x) & +\phi\left(x, K_{r} T x\right)+2\left\langle p-x, J x-J K_{r} T x\right\rangle \\
= & \phi\left(p, K_{r} T x\right) \\
\leq & \phi(p, T x)-\phi\left(K_{r} T x, T x\right) \\
\leq & \phi(p, x)+k \phi(x, T x)-\phi\left(K_{r} T x, T x\right) \\
\leq & (p, x)+2 \frac{k}{1-k}\langle x-p, J x-J T x\rangle-\phi\left(K_{r} T x, T x\right),
\end{aligned}
$$

and then

$$
\begin{aligned}
\phi(x, & \left.K_{r} T x\right)+\phi\left(K_{r} T x, T x\right) \\
& \leq 2 \frac{k}{1-k}\langle x-p, J x-J T x\rangle+2\left\langle x-p, J x-J K_{r} T x\right\rangle \\
& =2 \frac{k}{1-k}\langle x-p, J x-J T x\rangle+2\langle x-p, J x-J T x\rangle+2\left\langle x-p, J T x-J K_{r} T x\right\rangle \\
& =\left(2 \frac{k}{1-k}+2\right)\langle x-p, J x-J T x\rangle+2\left\langle x-p, J T x-J K_{r} T x\right\rangle \\
& =\frac{2}{1-k}\langle x-p, J x-J T x\rangle+2\left\langle x-p, J T x-J K_{r} T x\right\rangle .
\end{aligned}
$$

Lemma 2.11 (Alber and Reich [10]). Let $C$ be a nonempty closed convex subset of a smooth Banach space $E, x_{0} \in C$, and $x \in E$. Then, $x_{0}=\Pi_{C} x$ if and only if

$$
\left\langle x_{0}-y, J x-J x_{0}\right\rangle \geq 0, \quad \forall y \in C .
$$


Lemma 2.12 (Alber [30]). Let $E$ be a reflexive, strictly convex, and smooth Banach space, let $C$ be a nonempty closed convex subset of $E$, and let $x \in E$. Then

$$
\phi\left(y, \Pi_{C} x\right)+\phi\left(\Pi_{C} x, x\right) \leq \phi(y, x), \quad \forall y \in C .
$$

\section{Main Result}

Theorem 3.1. Let E be a reflexive, strictly convex, and smooth Banach space such that $E$ and $E^{*}$ have the property $(K)$. Assume that $C$ is a nonempty closed convex subset of $E$. Let $T: C \rightarrow C$ be a closed and $\phi$-quasi-strict pseudocontraction, $\Theta$ a bifunction from $C \times C$ to $\mathbb{R}$ satisfying (A1)-(A4), $\varphi: C \rightarrow \mathbb{R}$ a lower semi-continuous and convex function, and $A: C \rightarrow E^{*}$ a continuous and monotone mapping such that $\Omega:=F(T) \cap \operatorname{GMEP}(\Theta, A, \varphi) \neq \emptyset$. Define a sequence $\left\{x_{n}\right\}$ in $C$ by the following algorithm:

$$
\begin{aligned}
& x_{0} \in E \text {, chosen arbitrarily, } \\
& C_{1}=C, \\
& x_{1}=\Pi_{C_{1}}\left(x_{0}\right), \\
& u_{n} \in C \text { such that } \\
& \quad \Theta\left(u_{n}, y\right)+\left\langle A u_{n}, y-u_{n}\right\rangle+\varphi(y)-\varphi\left(u_{n}\right)+\frac{1}{r_{n}}\left\langle y-u_{n}, J u_{n}-J T x_{n}\right\rangle \geq 0, \quad \forall y \in C, \\
& C_{n+1}=\left\{z \in C_{n}: \phi\left(x_{n}, u_{n}\right)+\phi\left(u_{n}, T x_{n}\right)\right. \\
& \left.\quad \leq \frac{2}{1-k}\left\langle x_{n}-z, J x_{n}-J T x_{n}\right\rangle+2\left\langle x_{n}-z, J T x_{n}-J u_{n}\right\rangle\right\}, \\
& x_{n+1}=\prod_{C_{n+1}}\left(x_{0}\right),
\end{aligned}
$$

where $k \in[0,1)$ and $r_{n}>0$ for all $n \in \mathbb{N}$ with $\liminf _{n \rightarrow \infty} r_{n}>0$. Then, $\left\{x_{n}\right\}$ converges strongly to $\Pi_{\Omega}\left(x_{0}\right)$.

Proof. The proof is divided into seven steps.

Step 1. Show that $\Omega$ is closed and convex.

From step 1 of Zhou and Gao [25], $F(T)$ is closed and convex and by Lemma 2.9(4) $\operatorname{GMEP}(\Theta, A, \varphi)$ is closed and convex. So, $\Omega:=F(T) \cap \operatorname{GMEP}(\Theta, A, \varphi)$ is closed and convex.

Step 2. Show that $C_{n}$ is closed and convex for all $n \geq 1$.

For $n=1, C_{1}=C$ is closed and convex. Assume that $C_{k}$ is closed and convex for some $k \in \mathbb{N}$. For $z \in C_{k+1}$, one obtains that

$$
\phi\left(x_{k}, u_{k}\right)+\phi\left(u_{k}, T x_{k}\right) \leq \frac{2}{1-k}\left\langle x_{k}-z, J x_{k}-J T x_{k}\right\rangle+2\left\langle x_{k}-z, J T x_{k}-J u_{k}\right\rangle .
$$

It is not hard to see that the continuity and linearity of $\left\langle\cdot, J x_{k}-J T x_{k}\right\rangle$ and $\left\langle\cdot, J T x_{k}-J u_{k}\right\rangle$ allow $C_{k+1}$ to be closed and convex. Then, for all $n \geq 1, C_{n}$ is closed and convex.

Step 3. Show that $\Omega \subset C_{n}$ for all $n \geq 1$. 
It is obvious that $\Omega:=F(T) \cap \operatorname{GMEP}(\Theta, A, \varphi) \subset C=C_{1}$. Suppose that $\Omega \subset C_{k}$ for some $k \in \mathbb{N}$. For any $p \in \Omega$, we have $p \in C_{k}$. Notice that $x_{k}=\Pi_{C_{k}}\left(x_{0}\right)$, and then by Lemma 2.9 we obtain $u_{k}=K_{r_{k}} T x_{k}$. So, it follows from Lemma 2.10 that

$$
\phi\left(x_{k}, u_{k}\right)+\phi\left(u_{k}, T x_{k}\right) \leq \frac{2}{1-k}\left\langle x_{k}-p, J x_{k}-J T x_{k}\right\rangle+2\left\langle x_{k}-p_{,} J T x_{k}-J u_{k}\right\rangle .
$$

This means that $p \in C_{k+1}$. By mathematical induction, $\Omega \subset C_{n}$ for all $n \geq 1$. Therefore, $\Omega \subset$ $\bigcap_{n=1}^{\infty} C_{n}=: D \neq \emptyset$.

Step 4. Show that $\lim _{n \rightarrow \infty} \phi\left(x_{n}, x_{0}\right)$ exists.

By $x_{n}=\Pi_{C_{n}} x_{0}$ and Lemma 2.12, we have

$$
\phi\left(x_{n}, x_{0}\right)=\phi\left(\Pi_{C_{n}} x_{0}, x_{0}\right) \leq \phi\left(w, x_{0}\right)-\phi\left(w, x_{n}\right) \leq \phi\left(w, x_{0}\right),
$$

for each $w \in \Omega \subset C_{n}$ and for all $n \geq 1$. Therefore, the sequence $\left\{\phi\left(x_{n}, x_{0}\right)\right\}$ is bounded.

On the other hand, noticing that $x_{n}=\Pi_{C_{n}} x_{0}$ and $x_{n+1}=\Pi_{C_{n+1}} x_{0} \in C_{n+1} \subset C_{n}$, $\phi\left(x_{n}, x_{0}\right)=\min _{z \in C_{n}} \phi\left(z, x_{0}\right) \leq \phi\left(x_{n+1}, x_{0}\right)$ for all $n \geq 1$. Therefore, $\phi\left(x_{n}, x_{0}\right)$ is nondecreasing. It follows that the limit of $\phi\left(x_{n}, x_{0}\right)$ exists.

Step 5. Show that $x_{n} \rightarrow q$ as $n \rightarrow \infty$, where $q=\Pi_{D} x_{0}$.

Since $x_{n+1}=\Pi_{C_{n+1}} x_{0} \in C_{n+1} \subset C_{n}$ for any positive integer $n$ and by Lemma 2.12, we have

$$
\phi\left(x_{n+1}, x_{n}\right)=\phi\left(x_{n+1}, \Pi_{C_{n}} x_{0}\right) \leq \phi\left(x_{n+1}, x_{0}\right)-\phi\left(\Pi_{C_{n}} x_{0}, x_{0}\right)=\phi\left(x_{n+1}, x_{0}\right)-\phi\left(x_{n}, x_{0}\right) .
$$

Letting $n \rightarrow \infty$ in (3.5), one has $\phi\left(x_{n+1}, x_{n}\right) \rightarrow 0$ as $n \rightarrow \infty$. Without loss of generality, we can assume that $x_{n} \rightarrow q$ weakly as $n \rightarrow \infty$ (passing to a subsequence if necessary). It is easy to show that $q \in C_{n}$ for all $n \geq 1$. Hence $q \in \bigcap_{n=1}^{\infty} C_{n}=D$. Noticing that $\phi\left(x_{n}, x_{0}\right) \leq$ $\phi\left(x_{n+1}, x_{0}\right) \leq \phi\left(q, x_{0}\right)$, we have

$$
\left(q, x_{0}\right) \leq \liminf _{n \rightarrow \infty} \phi\left(x_{n}, x_{0}\right) \leq \limsup _{n \rightarrow \infty} \phi\left(x_{n}, x_{0}\right) \leq \phi\left(q, x_{0}\right)
$$

which implies that $\phi\left(x_{n}, x_{0}\right) \rightarrow \phi\left(q, x_{0}\right)$ as $n \rightarrow \infty$. Hence $\left\|x_{n}\right\| \rightarrow\|q\|$. By the property $(K)$ of $E$, we have $x_{n} \rightarrow q$. From Lemma 2.11, we have

$$
\left\langle x_{n}-y_{,} J x_{0}-J x_{n}\right\rangle \geq 0, \quad \forall y \in D
$$

Hence

$$
\left\langle q-y, J x_{0}-J q\right\rangle \geq 0, \quad \forall y \in D
$$

which implies that $q=\Pi_{D} x_{0}$.

Step 6. Show that $q \in \Omega$. 
We prove first that $\left\{T x_{n}\right\}$ and $\left\{u_{n}\right\}=\left\{K_{r_{n}} T x_{n}\right\}$ are bounded. Indeed, taking $p \in \Omega=$ $F(T) \cap \operatorname{GMEP}(\Theta, A, \varphi) \subset C_{n+1}$, we have

$$
\begin{aligned}
\|p\|^{2} & -2\|p\|\left\|T x_{n}\right\|+\left\|T x_{n}\right\|^{2} \\
& =\left(\|p\|-\left\|T x_{n}\right\|\right)^{2} \leq \phi\left(p, T x_{n}\right) \\
& \leq \phi\left(p, x_{n}\right)+k \phi\left(x_{n}, T x_{n}\right) \\
& \leq \phi\left(p, x_{n}\right)+\frac{2 k}{1-k}\left\langle x_{n}-p, J x_{n}-J T x_{n}\right\rangle \\
& \leq \phi\left(p, x_{n}\right)+\frac{2 k}{1-k}\left\|x_{n}-p\right\|\left\|x_{n}\right\|+\frac{2 k}{1-k}\left\|x_{n}-p\right\|\left\|T x_{n}\right\| .
\end{aligned}
$$

Then

$$
\begin{aligned}
\left\|T x_{n}\right\|^{2} & \leq\left(\phi\left(p, x_{n}\right)-\|p\|^{2}+\frac{2 k}{1-k}\left\|x_{n}-p\right\|\left\|x_{n}\right\|\right)+\left(\frac{2 k}{1-k}\left\|x_{n}-p\right\|+2\|p\|\right)\left\|T x_{n}\right\| \\
& \leq M+K\left\|T x_{n}\right\|=M+\frac{1}{2}\left(2 K\left\|T x_{n}\right\|\right) \\
& \leq M+\frac{1}{2}\left(K^{2}+\left\|T x_{n}\right\|^{2}\right)=M+\frac{1}{2} K^{2}+\frac{1}{2}\left\|T x_{n}\right\|^{2},
\end{aligned}
$$

where $M:=\sup \left\{\phi\left(p, x_{n}\right)-\|p\|^{2}+(2 k /(1-k))\left\|x_{n}-p\right\|\left\|x_{n}\right\|: n \in \mathbb{N}\right\}$ and $K:=\sup \{(2 k /(1-$ k)) $\left.\left\|x_{n}-p\right\|+2\|p\|: n \in \mathbb{N}\right\}$. Thus

$$
\left\|T x_{n}\right\|^{2} \leq 2 M+K^{2}
$$

for all $n \in \mathbb{N}$. Therefore $\left\{T x_{n}\right\}$ is bounded. Note that $\phi\left(p, K_{r_{n}} T x_{n}\right) \leq \phi\left(p, T x_{n}\right)$ for all $n \in \mathbb{N}$. Therefore $\left\{u_{n}\right\}=\left\{K_{r_{n}} T x_{n}\right\}$ is also bounded. From $x_{n+1} \in C_{n+1}$, one has

$$
\phi\left(x_{n}, u_{n}\right)+\phi\left(u_{n}, T x_{n}\right) \leq \frac{2}{1-k}\left\langle x_{n}-x_{n+1}, J x_{n}-J T x_{n}\right\rangle+2\left\langle x_{n}-x_{n+1}, J T x_{n}-J u_{n}\right\rangle .
$$

By Step 5, we obtain that $x_{n+1}-x_{n} \rightarrow 0$. Taking limit on both sides of (3.12), we obtain $\phi\left(x_{n}, u_{n}\right)+\phi\left(u_{n}, T x_{n}\right) \rightarrow 0$ as $n \rightarrow \infty$. Noting that $0 \leq\left(\left\|x_{n}\right\|-\left\|u_{n}\right\|\right)^{2} \leq \phi\left(x_{n}, u_{n}\right), 0 \leq$ $\left(\left\|u_{n}\right\|-\left\|T x_{n}\right\|\right)^{2} \leq \phi\left(u_{n}, T x_{n}\right)$, and $\left\|x_{n}\right\| \rightarrow\|q\|$, it is implied that

$$
\|q\|=\lim _{n \rightarrow \infty}\left\|x_{n}\right\|=\lim _{n \rightarrow \infty}\left\|u_{n}\right\|=\lim _{n \rightarrow \infty}\left\|T x_{n}\right\|,
$$

and consequently

$$
\|J q\|=\lim _{n \rightarrow \infty}\left\|J x_{n}\right\|=\lim _{n \rightarrow \infty}\left\|J u_{n}\right\|=\lim _{n \rightarrow \infty}\left\|J T x_{n}\right\| .
$$


This implies that $\left\{J\left(u_{n}\right)\right\}$ and $\left\{J\left(T x_{n}\right)\right\}$ are bounded. Since $E$ is reflexive, $E^{*}$ is also reflexive. So we can assume that

$$
J\left(u_{n}\right) \longrightarrow f \in E^{*}
$$

weakly. On the other hand, in view of the reflexivity of $E$, one has $J(E)=E^{*}$, which means that, for $f \in E^{*}$, there exists $x_{f} \in E$, such that $J x_{f}=f$. It follows that

$$
\phi\left(x_{n}, u_{n}\right)=\left\|x_{n}\right\|^{2}-2\left\langle x_{n}, J\left(u_{n}\right)\right\rangle+\left\|u_{n}\right\|^{2}=\left\|x_{n}\right\|^{2}-2\left\langle x_{n}, J\left(u_{n}\right)\right\rangle+\left\|J\left(u_{n}\right)\right\|^{2} .
$$

Taking $\lim \inf _{n \rightarrow \infty}$ on both sides of the equality above, we have

$$
\begin{aligned}
0 & \geq\|q\|^{2}-2\langle q, f\rangle+\|f\|^{2} \\
& =\|q\|^{2}-2\left\langle u_{0}, J x_{f}\right\rangle+\left\|J x_{f}\right\|^{2} \\
& =\phi\left(q, x_{f}\right) .
\end{aligned}
$$

Therefore $\phi\left(q, x_{f}\right)=0$ and consequently $q=x_{f}$, which implies that $f=J q$. Hence

$$
J\left(u_{n}\right) \longrightarrow J q \in E^{*}
$$

weakly. Since $\left\|J\left(u_{n}\right)\right\| \rightarrow\|J q\|$ and $E^{*}$ has the property $(K)$, we have

$$
\left\|J\left(u_{n}\right)-J q\right\| \longrightarrow 0
$$

Noting that $J^{-1}: E^{*} \rightarrow E$ is demicontinuous, we have

$$
u_{n} \longrightarrow q \in E
$$

weakly. Since $\left\|u_{n}\right\| \rightarrow\|q\|$ and $E$ has the property $(K)$, we obtain that $u_{n} \rightarrow q$ as $n \rightarrow \infty$. Similarly, it is not difficult to show that $T x_{n} \rightarrow q$ as $n \rightarrow \infty$. From $x_{n} \rightarrow q$ and the closeness property of $T$, we have $T q=q$.

Next, we want to show that $q \in \operatorname{GMEP}(\Theta, A, \varphi)$. Define $G: C \times C \rightarrow \mathbb{R}$ by $G(x, y)=$ $\Theta(x, y)+\langle A x, y-x\rangle+\varphi(y)-\varphi(x)$ for all $x, y \in C$. It is not hard to verify that $G$ satisfies conditions $(A 1)-(A 4)$. It follows from $u_{n}=K_{r_{n}} T x_{n}$ and $(A 2)$ that

$$
\frac{1}{r_{n}}\left\langle y-u_{n}, J u_{n}-J x_{n}\right\rangle \geq G\left(y, u_{n}\right), \quad \forall y \in C
$$

By using $(A 4)$ and $\liminf _{n \rightarrow \infty} r_{n}>0$, we obtain $0 \geq G(y, q)$ for all $y \in C$. For $t \in(0,1]$ and $y \in C$, let $y_{t}=t y+(1-t) q$. So, from $(A 1)$ and $(A 4)$, we have

$$
0=G\left(y_{t}, y_{t}\right)=G\left(y_{t}, t y+(1-t) q\right) \leq t G\left(y_{t}, y\right)+(1-t) G\left(y_{t}, q\right) \leq t G\left(y_{t}, y\right) .
$$


Dividing by $t$, we have

$$
G\left(y_{t}, y\right) \geq 0, \quad \forall y \in C
$$

From $(A 3)$ we have $0 \leq \lim _{t \rightarrow 0} G\left(y_{t}, y\right)=\lim _{t \rightarrow 0} G(t y+(1-t) q, y) \leq G(q, y)$ for all $y \in C$, and hence $q \in \operatorname{GMEP}(\Theta, A, \varphi)$. So, $q \in F(T) \cap \operatorname{GMEP}(\Theta, A, \varphi)=\Omega$.

Step 7. Show that $q=\Pi_{\Omega} x_{0}$.

It follows from Steps 5 and 6 that

$$
\phi\left(q, x_{0}\right) \leq \phi\left(\Pi_{\Omega} x_{0}, x_{0}\right) \leq \phi\left(q, x_{0}\right)
$$

which implies that $\phi\left(\Pi_{\Omega} x_{0}, x_{0}\right)=\phi\left(q, x_{0}\right)$. Hence, $q=\Pi_{\Omega} x_{0}$. Then $\left\{x_{n}\right\}$ converges strongly to $q=\Pi_{\Omega} x_{0}$. This completes the proof. corollary.

If $T$ is closed $\phi$-quasi-nonexpansive, then Theorem 3.1 is reduced to the following

Corollary 3.2. Let E be a reflexive, strictly convex, and smooth Banach space such that $E$ and $E^{*}$ have the property $(K)$. Assume that $C$ is a nonempty closed convex subset of $E$. Let $T: C \rightarrow C$ be a $\phi$ closed quasi-nonexpansive mapping, $\Theta$ a bifunction from $C \times C$ to $\mathbb{R}$ satisfying $(A 1)-(A 4), \varphi: C \rightarrow \mathbb{R}$ a lower semi-continuous and convex function, and $A: C \rightarrow E^{*}$ a continuous and monotone mapping such that $\Omega:=F(T) \cap \operatorname{GMEP}(\Theta, A, \varphi) \neq \emptyset$. Define a sequence $\left\{x_{n}\right\}$ in $C$ by the following algorithm:

$$
\begin{aligned}
& x_{0} \in E, \text { chosen arbitrarily, } \\
& C_{1}=C, \\
& x_{1}=\Pi_{C_{1}}\left(x_{0}\right), \\
& u_{n} \in C \text { such that } \\
& \quad \Theta\left(u_{n}, y\right)+\left\langle A u_{n}, y-u_{n}\right\rangle+\varphi(y)-\varphi\left(u_{n}\right)+\frac{1}{r_{n}}\left\langle y-u_{n}, J u_{n}-J T x_{n}\right\rangle \geq 0, \forall y \in C, \\
& C_{n+1}=\left\{z \in C_{n}: \phi\left(x_{n}, u_{n}\right)+\phi\left(u_{n}, T x_{n}\right) \leq 2\left\langle x_{n}-z, J x_{n}-J u_{n}\right\rangle\right\}, \\
& x_{n+1}=\Pi_{C_{n+1}}\left(x_{0}\right),
\end{aligned}
$$

where $r_{n}>0$ for all $n \in \mathbb{N}$ with $\lim _{\inf } f_{n \rightarrow \infty} r_{n}>0$. Then $\left\{x_{n}\right\}$ converges strongly to $\Pi_{\Omega}\left(x_{0}\right)$.

If $E=H$ is a Hilbert space, then Theorem 3.1 is reduced to the following corollary.

Corollary 3.3. Let $H$ be a Hilbert space. Assume that $C$ is a nonempty closed convex subset of $H$. Let $T: C \rightarrow C$ be a closed and $\phi$-quasi-strict pseudocontraction, $\Theta$ a bifunction from $C \times C$ to $\mathbb{R}$ satisfying (A1)-(A4), $\varphi: C \rightarrow \mathbb{R}$ a lower semi-continuous and convex function, and $A: C \rightarrow H$ 
a continuous and monotone mapping such that $\Omega:=F(T) \cap \operatorname{GMEP}(\Theta, A, \varphi) \neq \emptyset$. Define a sequence $\left\{x_{n}\right\}$ in $C$ by the following algorithm:

$$
\begin{aligned}
& x_{0} \in H \text {, chosen arbitrarily, } \\
& C_{1}=C, \\
& x_{1}=\prod_{C_{1}}\left(x_{0}\right), \\
& u_{n} \in C \text { such that, } \\
& \quad \Theta\left(u_{n}, y\right)+\left\langle A u_{n}, y-u_{n}\right\rangle+\varphi(y)-\varphi\left(u_{n}\right)+\frac{1}{r_{n}}\left\langle y-u_{n}, u_{n}-T x_{n}\right\rangle \geq 0, \quad \forall y \in C, \\
& C_{n+1}=\left\{z \in C_{n}:\left\|x_{n}-u_{n}\right\|^{2}+\left\|u_{n}-T x_{n}\right\|^{2}\right. \\
& \left.\quad \leq \frac{2}{1-k}\left\langle x_{n}-z, x_{n}-T x_{n}\right\rangle+2\left\langle x_{n}-z, T x_{n}-u_{n}\right\rangle\right\}, \\
& x_{n+1}=\prod_{C_{n+1}}\left(x_{0}\right),
\end{aligned}
$$

where $k \in[0,1)$ and $r_{n}>0$ for all $n \in \mathbb{N}$ with $\liminf _{n \rightarrow \infty} r_{n}>0$. Then $\left\{x_{n}\right\}$ converges strongly to $\Pi_{\Omega}\left(x_{0}\right)$.

$$
\text { If } A=0 \text { and } \varphi=0 \text {, then we have the following corollary. }
$$

Corollary 3.4. Let $E$ be a reflexive, strictly convex, and smooth Banach space such that $E$ and $E^{*}$ have the property $(K)$. Assume that $C$ is a nonempty closed convex subset of $E$. Let $T: C \rightarrow C$ be a closed and $\phi$-quasi-strict pseudocontraction and $\Theta$ a bifunction from $C \times C$ to $\mathbb{R}$ satisfying (A1)-(A4) such that $\Lambda:=F(T) \cap \mathrm{EP}(\Theta) \neq \emptyset$. Define a sequence $\left\{x_{n}\right\}$ in $C$ by the following algorithm:

$$
\begin{aligned}
& x_{0} \in \text { E, chosen arbitrarily, } \\
& C_{1}=C, \\
& x_{1}=\Pi_{C_{1}}\left(x_{0}\right), \\
& u_{n} \in C \text { such that } \\
& \quad \Theta\left(u_{n}, y\right)+\frac{1}{r_{n}}\left\langle y-u_{n}, J u_{n}-J T x_{n}\right\rangle \geq 0 \quad \forall y \in C, \\
& C_{n+1}=\left\{z \in C_{n}: \phi\left(x_{n}, u_{n}\right)+\phi\left(u_{n}, T x_{n}\right)\right. \\
& \left.\quad \leq \frac{2}{1-k}\left\langle x_{n}-z, J x_{n}-J T x_{n}\right\rangle+2\left\langle x_{n}-z, J T x_{n}-J u_{n}\right\rangle\right\}, \\
& x_{n+1}=\prod_{C_{n+1}}\left(x_{0}\right),
\end{aligned}
$$

where $k \in[0,1)$ and $r_{n}>0$ for all $n \in \mathbb{N}$ with $\liminf _{n \rightarrow \infty} r_{n}>0$. Then $\left\{x_{n}\right\}$ converges strongly to $\Pi_{\Omega}\left(x_{0}\right)$. 
Corollary 3.5 ([25, Theorem 3.1]). Let $E$ be a reflexive, strictly convex and smooth Banach space such that $E$ and $E^{*}$ have the property $(K)$. Assume that $C$ is a nonempty closed convex subset of $E$. Let $T: C \rightarrow C$ be a $\phi$-closed quasi-strict pseudocontraction. Define a sequence $\left\{x_{n}\right\}$ in $C$ by the following algorithm:

$$
\begin{aligned}
& x_{0} \in E, \text { chosen arbitrarily, } \\
& C_{1}=C, \\
& x_{1}=\Pi_{C_{1}}\left(x_{0}\right), \\
& u_{n} \in C \text { such that } \\
& C_{n+1}=\left\{z \in C_{n}: \phi\left(x_{n}, T x_{n}\right) \leq \frac{2}{1-k}\left\langle x_{n}-z, J x_{n}-J T x_{n}\right\rangle\right\}, \\
& x_{n+1}=\Pi_{C_{n+1}}\left(x_{0}\right)
\end{aligned}
$$

where $k \in[0,1)$. Then $\left\{x_{n}\right\}$ converges strongly to $\Pi_{\Omega}\left(x_{0}\right)$.

Proof. Put $\Theta=0, A=0, \varphi=0$, and $r_{n}=1$ for all $n \geq 1$ in Theorem 3.1. Then, $K_{r_{n}}=\Pi_{C}$ for all $n \geqslant 1$. So, $u_{n}=\Pi_{C} T x_{n}$ for all $n \geqslant 1$ (note that $x_{1}=\Pi_{C} x_{0}$ ). Since $x_{n}=\Pi_{C_{n}} x_{0} \in C_{n} \subset C$ and then $T x_{n} \in C$ for all $n \geqslant 1$, we have $u_{n}=T x_{n}$ for all $n \geqslant 1$. Thus $\phi\left(x_{n}, u_{n}\right)+\phi\left(u_{n}, T x_{n}\right)=\phi\left(x_{n}, T x_{n}\right)$ and $J T x_{n}-J u_{n}=0$ for all $n \geq 1$. For this reason, (1.12) is a special case of (3.1). Applying Theorem 3.1, we have the desired result.

Remark 3.6. It is well known that every uniformly convex and uniformly smooth Banach space satisfies all assumptions of Banach space in Theorem 3.1. On the other hand, in general, Musielak-Orlicz space [26] need not be uniformly convex or uniformly smooth, however, any strictly convex, reflexive and smooth Musielak-Orlicz space [26] satisfies all Banach-space assumptions of Theorem 3.1. It can be written as the following diagram:

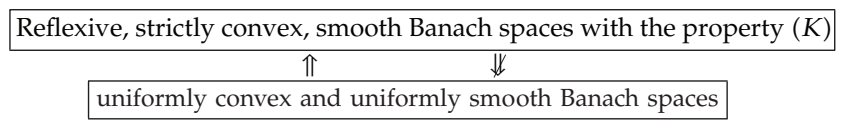

For this reason, Theorem 3.1 can be viewed as a more general one and can be applied widely in both the fixed point problems and the equilibrium problems.

\section{Acknowledgments}

The author is supported by Thailand Research Fund under Grant no. MRG5380249 and the Centre of Excellence in Mathematics under the Commission on Higher Education, Ministry of Education, Thailand. The author would like to thank Simeon Reich and an anonymous referee for their valuable comments and suggestions, which were helpful in improving the paper. 


\section{References}

[1] W. R. Mann, "Mean value methods in iteration," Proceedings of the American Mathematical Society, vol. 4, pp. 506-510, 1953.

[2] H. H. Bauschke, E. Matoušková, and S. Reich, "Projection and proximal point methods: convergence results and counterexamples," Nonlinear Analysis, vol. 56, no. 5, pp. 715-738, 2004.

[3] S. Reich, "Weak convergence theorems for nonexpansive mappings in Banach spaces," Journal of Mathematical Analysis and Applications, vol. 67, no. 2, pp. 274-276, 1979.

[4] K. Nakajo and W. Takahashi, "Strong convergence theorems for nonexpansive mappings and nonexpansive semigroups," Journal of Mathematical Analysis and Applications, vol. 279, no. 2, pp. 372379, 2003.

[5] T.-H. Kim and H.-K. Xu, "Strong convergence of modified Mann iterations for asymptotically nonexpansive mappings and semigroups," Nonlinear Analysis, vol. 64, no. 5, pp. 1140-1152, 2006.

[6] G. Marino and H.-K. Xu, "Weak and strong convergence theorems for strict pseudo-contractions in Hilbert spaces," Journal of Mathematical Analysis and Applications, vol. 329, no. 1, pp. 336-346, 2007.

[7] C. Martinez-Yanes and H.-K. Xu, "Strong convergence of the CQ method for fixed point iteration processes," Nonlinear Analysis, vol. 64, no. 11, pp. 2400-2411, 2006.

[8] S. Plubtieng and K. Ungchittrakool, "Strong convergence of modified Ishikawa iteration for two asymptotically nonexpansive mappings and semigroups," Nonlinear Analysis, vol. 67, no. 7, pp. 23062315, 2007.

[9] W. Takahashi, Y. Takeuchi, and R. Kubota, "Strong convergence theorems by hybrid methods for families of nonexpansive mappings in Hilbert spaces," Journal of Mathematical Analysis and Applications, vol. 341, no. 1, pp. 276-286, 2008.

[10] Y. I. Alber, "Metric and generalized projection operators in Banach spaces: properties and applications," in Theory and Applications of Nonlinear Operators of Accretive and Monotone Type, A. Kartsatos, Ed., pp. 15-50, Marcel Dekker, New York, NY, USA, 1996.

[11] S. Kamimura and W. Takahashi, "Strong convergence of a proximal-type algorithm in a Banach space," SIAM Journal on Optimization, vol. 13, no. 3, pp. 938-945, 2002.

[12] S. Reich, "A weak convergence theorem for the alternating method with Bregman distances," in Theory and Applications of Nonlinear Operators of Accretive and Monotone Type, A. G. Kartsatos, Ed., pp. 313-318, Marcel Dekker, New York, NY, USA, 1996.

[13] D. Butnariu, S. Reich, and A. J. Zaslavski, "Asymptotic behavior of relatively nonexpansive operators in Banach spaces," Journal of Applied Analysis, vol. 7, no. 2, pp. 151-174, 2001.

[14] D. Butnariu, S. Reich, and A. J. Zaslavski, "Weak convergence of orbits of nonlinear operators in reflexive Banach spaces," Numerical Functional Analysis and Optimization, vol. 24, no. 5-6, pp. 489-508, 2003.

[15] Y. Censor and S. Reich, "Iterations of paracontractions and firmly nonexpansive operators with applications to feasibility and optimization," Optimization, vol. 37, no. 4, pp. 323-339, 1996.

[16] S. Matsushita and W. Takahashi, "A strong convergence theorem for relatively nonexpansive mappings in a Banach space," Journal of Approximation Theory, vol. 134, no. 2, pp. 257-266, 2005.

[17] S. Plubtieng and K. Ungchittrakool, "Strong convergence theorems for a common fixed point of two relatively nonexpansive mappings in a Banach space," Journal of Approximation Theory, vol. 149, no. 2, pp. 103-115, 2007.

[18] S. Plubtieng and K. Ungchittrakool, "Strong convergence theorems of block iterative methods for a finite family of relatively nonexpansive mappings in Banach spaces," Journal of Nonlinear and Convex Analysis, vol. 8, no. 3, pp. 431-450, 2007.

[19] E. Blum and W. Oettli, "From optimization and variational inequalities to equilibrium problems," The Mathematics Student, vol. 63, no. 1-4, pp. 123-145, 1994.

[20] S. Takahashi and W. Takahashi, "Viscosity approximation methods for equilibrium problems and fixed point problems in Hilbert spaces," Journal of Mathematical Analysis and Applications, vol. 331, no. 1, pp. 506-515, 2007.

[21] A. Tada and W. Takahashi, "Strong convergence theorem for an equilibrium problem and a nonexpansive mapping," in Nonlinear Analysis and Convex Analysis, W. Takahashi and T. Tanaka, Eds., pp. 609-617, Yokohama Publishers, Yokohama, Japan, 2007.

[22] A. Tada and W. Takahashi, "Weak and strong convergence theorems for a nonexpansive mapping and an equilibrium problem," Journal of Optimization Theory and Applications, vol. 133, no. 3, pp. 359-370, 2007. 
[23] W. Takahashi and K. Zembayashi, "Strong convergence theorem by a new hybrid method for equilibrium problems and relatively nonexpansive mappings," Fixed Point Theory and Applications, vol. 2008, Article ID 528476, 11 pages, 2008.

[24] W. Takahashi and K. Zembayashi, "Strong and weak convergence theorems for equilibrium problems and relatively nonexpansive mappings in Banach spaces," Nonlinear Analysis, vol. 70, no. 1, pp. 45-57, 2009.

[25] H. Zhou and X. Gao, "An iterative method of fixed points for closed and quasi-strict pseudo-contractions in Banach spaces," Journal of Applied Mathematics and Computing, vol. 33, no. 1-2, pp. 227-237, 2010.

[26] H. Hudzik, W. Kowalewski, and G. Lewicki, "Approximate compactness and full rotundity in Musielak-Orlicz spaces and Lorentz-Orlicz spaces," Zeitschrift für Analysis und ihre Anwendungen, vol. 25, no. 2, pp. 163-192, 2006.

[27] I. Cioranescu, Geometry of Banach Spaces, Duality Mappings and Nonlinear Problems, Kluwer Academic Publishers, Dodrecht, The Netherlands, 1990.

[28] S. Reich, "Book review: geometry of Banach spaces, duality mappings and nonlinear problems by loana Cioranescu, Kluwer Academic Publishers, Dordrecht, 1990," Bulletin of the American Mathematical Society, vol. 26, no. 2, pp. 367-370, 1992.

[29] W. Takahashi, Nonlinear Functional Analysis, Yokohama Publishers, 2000.

[30] Y. I. Alber and S. Reich, "An iterative method for solving a class of nonlinear operator equations in Banach spaces," Panamerican Mathematical Journal, vol. 4, no. 2, pp. 39-54, 1994.

[31] S.-S. Zhang, "Generalized mixed equilibrium problem in Banach spaces," Applied Mathematics and Mechanics, vol. 30, no. 9, pp. 1105-1112, 2009. 


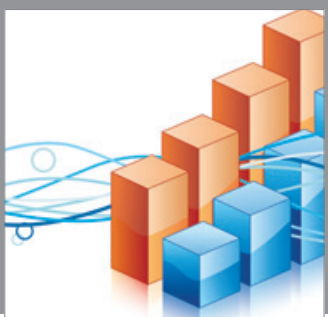

Advances in

Operations Research

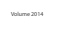

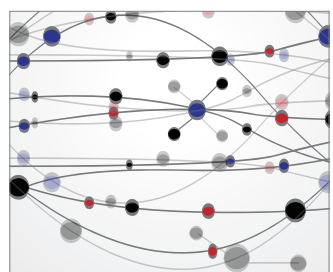

\section{The Scientific} World Journal
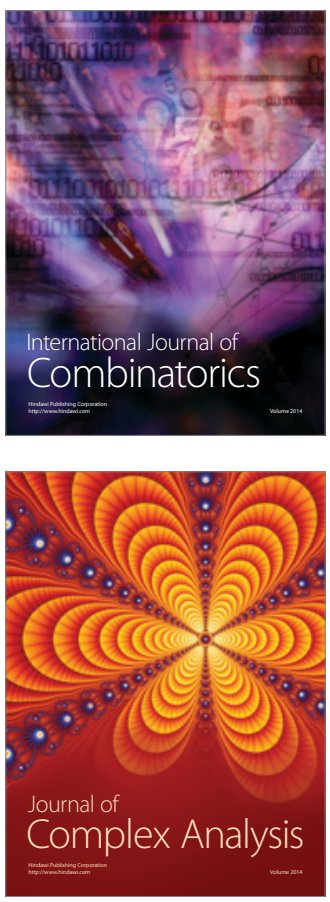

International Journal of

Mathematics and

Mathematical

Sciences
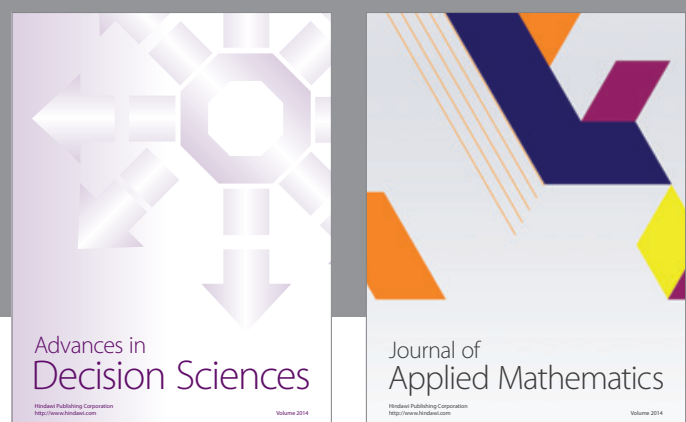

Journal of

Applied Mathematics
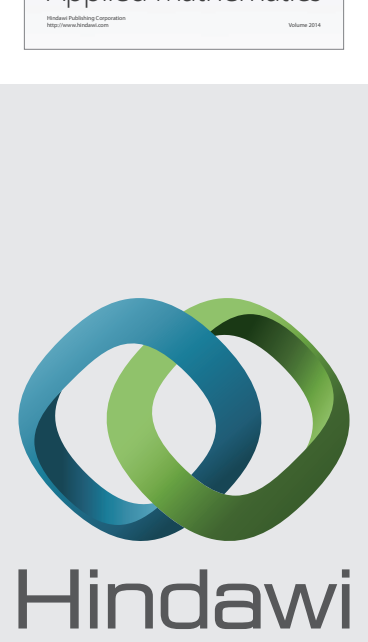

Submit your manuscripts at http://www.hindawi.com
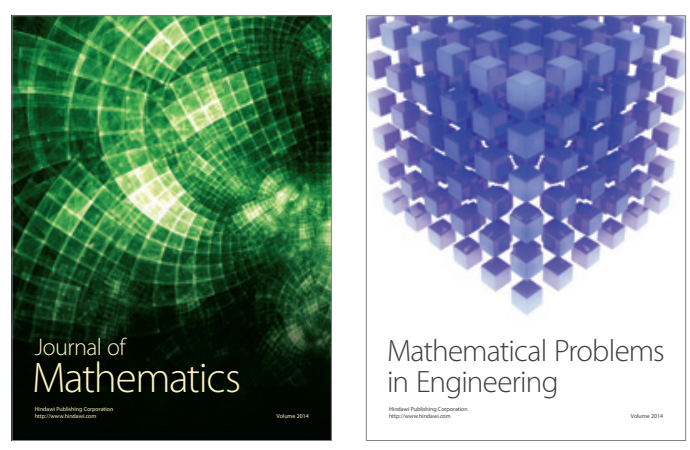

Mathematical Problems in Engineering
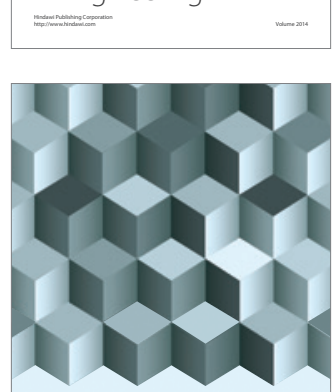

Journal of

Function Spaces
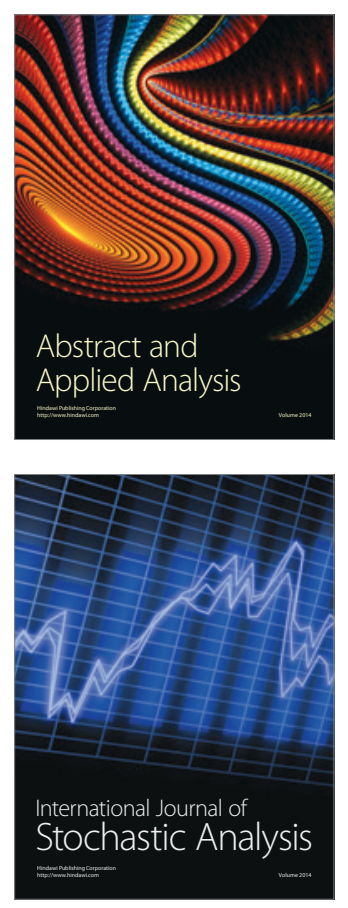

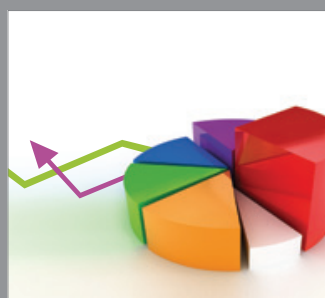

ournal of

Probability and Statistics

Promensencen
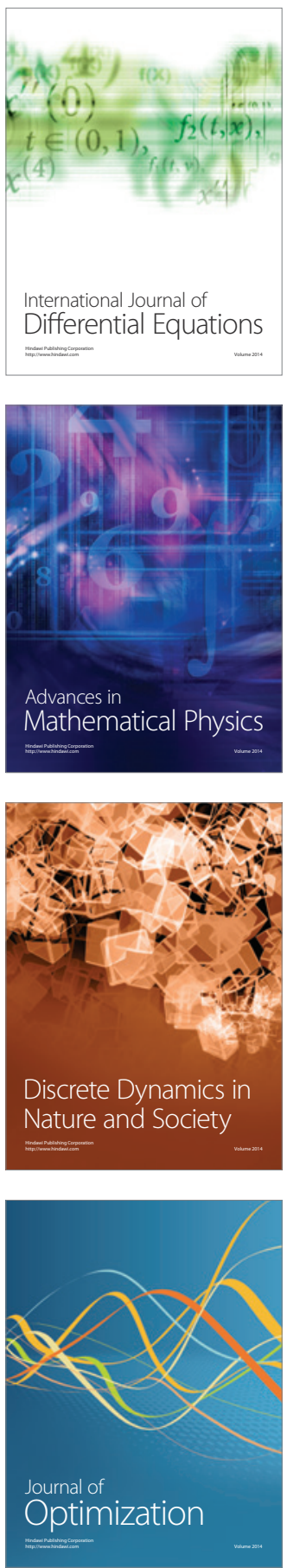